scattered sky light. Although the instrument behaves fairly well, its drive and clamping mechanism need a thorough overhaul; the dome does not turn very freely, and the axles and bearings of the wheels carrying it are badly worn and will soon require renewing. The 7-in. telescope, now that the "Bright Star Programme" is finished, is used for observing occultations, and also by Mr. W. P. Hirst, a local amateur, for the micrometric measurement of double stars ; Mr. A. G. F. Morrisby also uses it for observing variable stars. The 6-in. telescope, still utilized by visitors and for observing occultations, has been used regularly by Mr. R. P. de Kock for observing longperiod variable stars. The Tower telescope and the photoheliograph, as described in the 1951 report, continue to be used, the first for observing occulta. tions and variable stars, and the second for taking photographs-two each day-of the sun. A new photoelectric photometer, which was built during 1952 and used for a time on the Victoria telescope, has been transferred to the astrographic telescope to replace the old and rather inefficient photometer that was built originally for the 7-in. telescope ; this new photometer will be used for the "Bright Star Programme" already referred to.

Nearly half the report is included under the heading "Observations and Reductions", and comprises : meridian observations, photographic star positions and proper motions, the parallax programme, Radcliffe section, stellar photometry, photoheliograph, occultations and variable stars. Under each of these, full accounts are given of the progress during the year under consideration. In the section on stellar photometry, reference is made to the photometric cameras, which were not mounted on the astrographic refractor until November 1952, and were brought into use in December. An outline of the two short programmes for this instrument is given, the second of which is required to complete the work done at the University of Cambridge Observatories.

A reorganization of the time service and clock installation was begun in August ; but much detailed work still remains to be done, although the major changes were completed by the end of 1952. All the telephone cables in the grounds have been completely renewed by the G.P.O., to whose "remarkable speed, efficiency and close cooperation throughout, the Observatory is deeply indebted", in the words of the director. The Observatory keeps a limited stock of chronometers and watches for issue to H.M. ships ; in 1952 nine were issued and ten received. Meteorological work proceeded on the usual lines, continuous records being obtained of barometric pressure, temperature, humidity, wind direction and wind velocity on self-registering instruments.

Towards the end of the report reference is made to the jubilee meeting of the South African Association for the Advancement of Science, which was held in July and which brought a number of distinguished scientists to Cape Town. After the meeting, Prof. J. H. Oort gave a popular lecture under the auspices of the Board of Extra-Mural Studies of the University of Cape Town, and so great was the popular response to this lecture that, by request of the University, Dr. D. S. Evans gave a series of six evening lectures on modern astronomical problems, which were all very well attended. Earlier in the year an astronomical exhibit, organized by Dr. Evans, was shown in the FIall of Science at the van Riebeeck Festival Fair.
It is satisfactory to know that, in addition to the regular visiting days and the opening of the Observatory for inspection one afternoon "a month, the 5-in. and 6-in. telescopes have been placed at the disposal of the Cape Centre of the Astronomical Society of South Africa for the entertainment of visitors. These evenings have proved so popular that there has always been a long waiting list for the admission permits.

\section{EUROPEAN TIMBER TRENDS AND STATISTICS}

TN two United Nations publications, "European Timber Trends and Prospects"* and "European Timber Statistics, 1913-1950" $\dagger$, the Food and Agriculture Organization of the United Nations has summarized information collected jointly by it and the Timber Committee of the Economic Commission of Europe; the second publication arises more or less out of the investigations contained in the first.

In the autumn of 1947, when the Timber Committee of the Economic Commission of Europe was first established, its purpose was to help European governments to overcome, through concerted action, the acute timber shortage which was hampering European reconstruction. To a certain extent success was achieved, despite the temporary disappearance of the U.S.S.R. as a large source of soft-wood supplies and without aggravating Europe's dollar deficit through continuous heavy imports of North American timber. By 1949 the temporary equilibrium reached was precarious and obviously unstable, and war restrictions in the consumption of timber and paper were still in force. But the questions still remained of whether or not Europe was still faced with a timber shortage, and how would supply and demand of forest products develop over a longer span of years. The Committee therefore undertook a study of Europern timber production, consumption and trade, primarily to find answers to these questions, and its findings are jointly summarized in "European Timber Trends and Prospects", which is a monumental piece of research work. The resulting beliefs appear to be that by 1960 Europe may be deficient in timber unless she increases her own production by a "dynamic forest policy" of reafforestation and so forth, or imports larger amounts from North America and the tropical forests. It is open to doubt, taking into consideration the considerable period of time it takes trees to reach timber size, whether long forecasts are possible.

In the accompanying volume on "European Timber Statistics, 1913-1950" the periods covering the war years 1914-19 and 1939-45 have been excluded owing to the lack of adequate statistical information about them. This may seem to lessen the value of the inquiry somewhat since excessive fellings were made in the forests during the two World Wars, fellings which reduced subsequent consumption for a number of years. The volume is divided into three sets of tables, dealing respectively with production, trade

* European Timber Trends and Prospects: a Study prepared jointly by the Secretariats of the Food and Agriculture Organization of the United Nations and the United Nations Economic Commission for Europe. Pp. xvi + 315. (Geneva: U.N.(F.A.
H.M.S.O., 1953.) 14 Swiss fr., 25s, or 3.50 dollars.

$\dagger$ European Timber Statistics, 1913-1950 : Statistiques Européenes $\mathrm{du}^{\dagger}$ European Timber Statistics, 1913-1950: Statistiques Européenes du Bois, 1913-1950. Pp. v+168. (Geneva
H.M.S.O., 1953.) 7 Swiss fr., 12s. $6 d$., or 1.75 dollars. 
and consumption. It is stated that the object of the investigation is an attempt to fill a need felt by the majority of those entrusted with the role of wood in the European economy or who have tried to grapple with the timber problem on a European scale. For the first time, it is claimed, an attempt has been made to arrive at figures for trade and production in wood and its products for every European country, and to bring them together in the form of a convenient reference. This aim appears to have been usefully achieved. The volume has an increased value as it is in both English and French, the former on the left-hand pages and the latter on the righta most effective method of increasing its utility.

E. P. Stebring

\section{PARAMAGNETIC RESONANCE IN NEUTRON-IRRADIATED DIAMOND AND SMOKY QUARTZ}

\section{By Dr. J. H. E. GRIFFITHS, J. OWEN and I. M. WARD \\ Clarendon Laboratory, Oxford}

$\mathrm{T}$ HE nature of lattice defects in neutron-irradiated diamond is a problem of current interest. These defects are known to cause changes in some of the physical properties ${ }^{1}$ and give rise to a paramagnetic absorption spectrum. We have measured this spectrum in the temperature-range $20^{\circ}-290^{\circ} \mathrm{K}$., using wave-lengths of $1 \cdot 2$ and $3 \cdot 1 \mathrm{~cm}$. There are many closely spaced lines, of which two main types can be distinguished.

(a) There is an isotropic absorption line with spectroscopic splitting factor $g=2 \cdot 0028 \pm 0 \cdot 0006$. Both the intensity and width increase with duration of irradiation, but are reduced after the diamond has been heated to about $1,000^{\circ} \mathrm{C}$. (The intensity of the coloration produced by the irradiation varies in a similar way.) The line width, which after long irradiation is of the order of 100 gauss, decreases with temperature, being approximately half as big at $90^{\circ} \mathrm{K}$. as at $290^{\circ} \mathrm{K}$. This type of absorption line has also been observed in diamonds coloured by electron irradiation.

(b) There is an anisotropic spectrum consisting of lines symmetrically arranged about the type $(a)$ line, and of the order a hundred times smaller in intensity. The intensity does not appear to be affected after her.ting to $1,000^{\circ} \mathrm{C}$., and the line width is independent of temperature, being of the order of 5 gauss. This structure has been investigated in several Type 1 and Type 2 diamonds where the large central line had been reduced by heating. It is found that the positions in the spectrum of the most intense twelve of these anisotropic lines are identical in all the specimens that we have examined. They appear to arise from paramagnetic units in the crystal with electronic spin $S=1$, the spin-levels being separated into a doublet and a singlet (separation $D=0.010$ $\mathrm{cm}^{-1}$ ) by a crystalline field of nearly axial symmetry. This axis is found to be parallel to one of the six edges of the fundamental carbon tetrahedron of the diamond lattice, so that there are six differently oriented units present, each giving two lines in the spectrum (see Fig. 1). The mean spectroseopic splitting factor is $g=2.0027 \pm 0.0005$, and has a very small anisotropy given by $g_{\perp} / g \|=1 \cdot 00035 \pm$

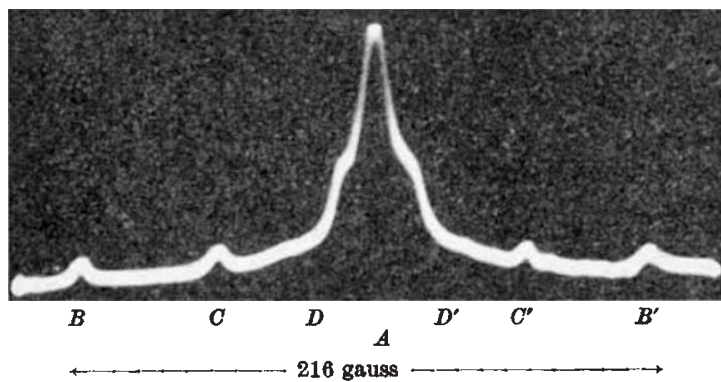

Fig. 1. Spectrum from an irradiated diamond with magnetic fleld $H$ parallel to $[1,1,0]$

$A$, central type (a) line (reduced by heating); $B$ and $B^{\prime}$ lines from one unit with axis parallel to $H$ (that is, parallel to $[1,1,0]$ ) $C$ and $C$, lines from one unit with axis perpendicular to $H, D$ and $D^{\prime}$, superimposed lines from four units with axes inclined at $60^{\circ}$ to $H$

$0 \cdot 00005$. For a general direction of the magnetic field the positions of the lines from each unit are given by the usual spin Hamiltonian $\mathcal{H}=\beta H . g . S+$ $D S_{z}^{2}$, the constants having the values given above. Apart from these twelve lines, other smaller lines are also present, the intensities and positions of which in the spectrum are not found to be the same in all specimens.

A preliminary theoretical investigation of the magnetic properties of neutron-irradiated diamond, in which it is assumed that carbon atoms are knocked into interstitial positions leaving vacancies in the lattice, has been made by Pryce ${ }^{2}$. Although it is too early to give any detailed interpretation of the spectrum described above, a tentative explanation which would give the correct spins of $S=1$ and the particular axes of distortion is that this spectrum arises from interstitial carbon atoms linked together in pairs.

The centres which are produced in quartz by $\mathrm{X}$-irradiation, giving rise to the well-known smoky colour, appear to be of a different kind from those in diamond, and have been thought for some time to be connected with the presence of impurity atoms in the silica lattice (see, for example, Brown and Thomas $\left.{ }^{8}\right)$. We have investigated the paramagnetic resonance in irradiated natural crystalline quartz and have found that a few hours irradiation by either neutrons, electrons or X-rays gives rise to the same spectrum. Prolonged irradiation by neutrons gives rise to additional absorption lines which will not be discussed here. For quartz irradiated by $X$-rays, it appears that the intensity of paramagnetic absorption is directly proportional to the coloration produced; the lines are completely removed by heating the quartz to $350^{\circ} \mathrm{C}$. The spectrum is also found in synthetic quartz, but is much reduced in intensity.

Measurements using wave-lengths of $1 \cdot 2$ and $3.1 \mathrm{~cm}$. have been made at $90^{\circ} \mathrm{K}$., the lines being very much broader at higher temperatures. The results indicate that there are six types of paramagnetic unit, each with electronic spin $S=\frac{1}{2}$ and the same principal $g$-values $\left(g_{\mid \mathrm{H}}=2 \cdot 06 \pm 0.005\right.$; $\left.g_{\perp}=2.00 \pm 0.005\right)$, but with differently oriented axes of magnetic symmetry. Each of the six absorption lines appears to be subdivided into six lines of roughly equal intensity with an overall separation of about 28 gauss at both wave-lengths (see Fig. 2). It is possible that this is a hyperfine structure, which suggests that the magnetic centres involve electrons, 